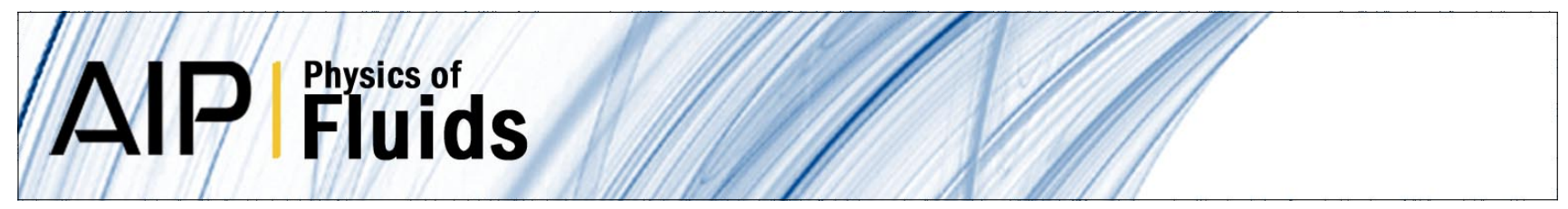

\title{
Buckling of a thin, viscous film in an axisymmetric geometry
}

S. Bhattacharya, R. V. Craster, and M. R. Flynn

Citation: Phys. Fluids 25, 043102 (2013); doi: 10.1063/1.4798825

View online: http://dx.doi.org/10.1063/1.4798825

View Table of Contents: http://pof.aip.org/resource/1/PHFLE6/v25/i4

Published by the American Institute of Physics.

\section{Additional information on Phys. Fluids}

Journal Homepage: http://pof.aip.org/

Journal Information: http://pof.aip.org/about/about_the_journal

Top downloads: http://pof.aip.org/features/most_downloaded

Information for Authors: http://pof.aip.org/authors

\section{ADVERTISEMENT}

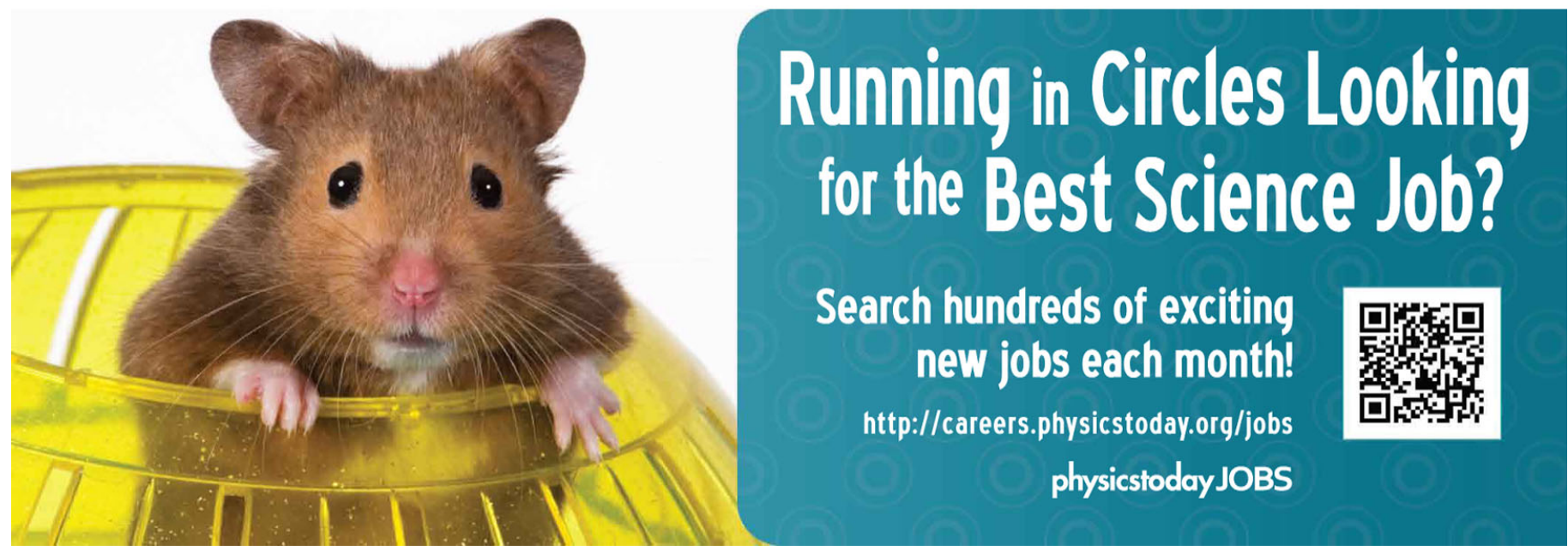




\title{
Buckling of a thin, viscous film in an axisymmetric geometry
}

\author{
S. Bhattacharya, ${ }^{1}$ R. V. Craster, ${ }^{2}$ and M. R. Flynn ${ }^{1}$ \\ ${ }^{1}$ Department of Mechanical Engineering, University of Alberta, Edmonton, Alberta T6G \\ 2G8, Canada \\ ${ }^{2}$ Department of Mathematics, Imperial College London, South Kensington Campus, London \\ SW7 2AZ, United Kingdom
}

(Received 28 May 2012; accepted 6 March 2013; published online 5 April 2013)

By adapting the Föppl-von Kàrmàn equation, which describes the deformation of a thin elastic membrane, we present an analysis of the buckling pattern of a thin, very viscous fluid layer subject to shear in an axisymmetric geometry. A linear stability analysis yields a differential eigenvalue problem, whose solution, obtained using spectral techniques, yields the most unstable azimuthal wave-number, $m^{\star}$. Contrary to the discussion of Slim et al. [J. Fluid Mech. 694, 5-28 (2012)], it is argued that the axisymmetric problem shares the same degeneracy as its rectilinear counterpart, i.e., at the onset of instability, $m^{\star}$ is indefinitely large. Away from this point, however, a comparison with analogue experimental results is both possible and generally favorable. In this vein, we describe the laboratory apparatus used to make new measurements of $m^{\star}$, the phase speed and the wave amplitude; note that no prediction concerning the latter two quantities can be made using the present theory. Experiments reveal a limited range of angular velocities wherein waves of either small or large amplitude may be excited. Transition from one to the other regime does not appear to be associated with a notable change in $m^{\star}$. ๑ 2013 American Institute of Physics. [http://dx.doi.org/10.1063/1.4798825]

\section{INTRODUCTION}

Thin viscous films floating on top of a much deeper and less viscous ambient fluid are a subject of topical scientific interest and appear in a multitude of industrial and environmental contexts. The Deepwater Horizon oil spill provides a familiar, if disastrously unfortunate, example. Although remediation efforts were multifaceted, it is very likely that a greater fraction of the spilt oil could have been recovered, had there existed a more comprehensive understanding of the rheology of the oil film relating, in particular, to the action of waves and wind shear, film rupture, the formation of "tar balls," etc.

The present investigation does not attempt to model the very complicated fluid mechanics associated with the calamitous events described above but rather considers a more fundamental problem, namely the regular pattern of surface waves that arises when an axisymmetric viscous film experiences buckling by shear forces. Part of our motivation is to add an important clarification to the recent paper by Slim, Teichman, and Mahadevan ${ }^{1}$ who examined theoretically and numerically the patterns of thin film buckling instabilities that arise due to in-plane shear in a rectilinear geometry (Figure 1(a)). Building on the analysis of Suleiman and Munson, ${ }^{2}$ Howell, ${ }^{3}$ Teichman, ${ }^{4}$ and Slim et al. ${ }^{5}$ Slim et al. ${ }^{1}$ introduce, as an alternative to (numerically) solving the full Stokes equations, a linear "viscous plate model" applicable in the limits where the film curvature and $h / \hat{L}$ are small. Here $h$ and $\hat{L}$ denote, respectively, the film thickness and a representative horizontal length scale. (For example, for the flows exhibited schematically in Figure 1, it is sensible to set $\hat{L}$ equal to the gap width.) The viscous plate model is itself similar, though not as we argue below completely analogous, to the classical Föppl-von Kàrmàn equation (Love, ${ }^{6}$ Landau and Lifshitz ${ }^{7}$ ), which describes the motion of an elastic membrane rather than a viscous film. Citing the Stokes-Rayleigh analogy, Slim 


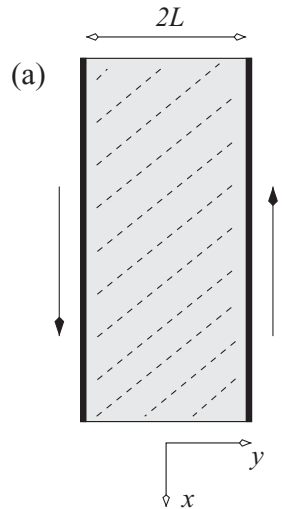

(b)

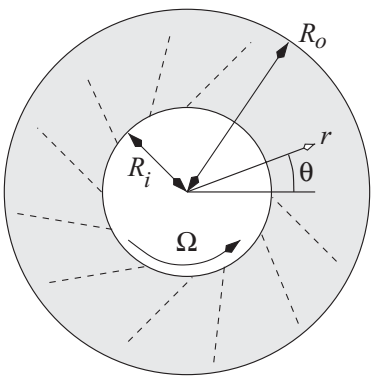

(c)

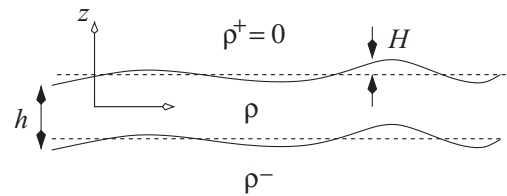

FIG. 1. Plan view of shear-driven buckling of a thin viscous film in (a) a rectilinear channel and (b) an axisymmetric channel. Troughs and crests of the associated wave field are indicated by the dashed lines. Panel (c) shows a side view of the thin film and its (sinuous) deflection from the horizontal where $\rho$ denotes the film fluid density.

et $a l .{ }^{1}$ remark that "there is a close connection between the governing equations for elastic and viscous plates as well as between the associated buckling instabilities."

Application of the Föppl-von Kàrmàn equation in the fluidic context has been criticized by Benjamin and Mullin ${ }^{8}$ who observed that the wavelength of the most unstable mode "is indefinitely small when the [linear] instability condition...is just satisfied." Moreover the stability threshold coincides, for reasons discussed below, with vanishingly small rather than finite forcing. Slim et al. ${ }^{1}$ acknowledge this inconsistency in the rectilinear case, but argue that it disappears in the axisymmetric flow of Figure 1(b), which includes as canonical horizontal length scales both the gap width and some measure of the curvature of the inner (rotating) or outer (stationary) cylinder. Herein, we argue that the axisymmetric flow in fact shares the same inconsistencies as its rectilinear counterpart. By considering the growth rates associated with the most unstable mode, however, we also argue that the degeneracy (whether viewed from an axisymmetric or a rectilinear perspective) is not so severe as to render the associated theory "primitive" to use the words of Benjamin and Mullin. ${ }^{8}$ Corroborating this discussion, our investigation is supplemented by new laboratory measurements of $m^{\star}$, the most unstable azimuthal wave-number, which show better agreement with theory than is reported in a previous analysis, i.e., Figures 3-5(a) and 3-7 of Teichman. ${ }^{4}$

The theory described above is, we reiterate, a linear theory applicable in the limit of small interfacial deflections. Although we do not extend our analysis to the weakly- or fully-nonlinear regime in the manner of, e.g., Balmforth, Craster, and Slim, ${ }^{9}$ we do report upon a further set of experiments which characterize, for select combinations of parameters, the variation of the wave amplitude as a function of the applied shear. Our measurements suggest a limited range of bi-stability in which waves of either small or large amplitude may arise for the same shear rate.

The rest of the paper is organized as follows: in Sec. II we summarize the viscous plate model as applied to the axisymmetric geometry of Figure 1(b). The problem is formulated in Sec. II A with some of the more technical details given in Appendices A and B. In Sec. II B, we summarize the most pertinent of our model predictions. A description of the experimental apparatus and procedure is given in Sec. III and a comparison between theory and experiment is presented thereafter in Sec. IV A. This is followed by a discussion of our wave amplitude measurements in Sec. IV B. Finally, in Sec. V, we present our conclusions and outline ideas for future work.

\section{THEORY}

\section{A. Formulation}

We consider the axisymmetric flow of a thin film of viscous fluid of thickness $h$ as depicted schematically in Figures 1(b) and 1(c). The film floats atop a deep layer of much less viscous fluid (water, say) whose density, $\rho^{-}$, is greater than the density, $\rho$, of the film. Out-of-plane displacements, 
$H$, are driven by the in-plane stresses associated with the rotating inner cylinder. The governing equations describing this free-surface problem read

$$
\begin{gathered}
\nabla \cdot \mathbf{u}=0, \\
\nabla P=\mu \nabla^{2} \mathbf{u},
\end{gathered}
$$

subject to a kinematic boundary condition

$$
\left.w\right|_{z=H \pm \frac{1}{2} h}=\frac{\mathrm{D}}{\mathrm{D} t}\left(H \pm \frac{1}{2} h\right)
$$

and a dynamic boundary condition

$$
\tau \cdot \mathbf{n}=\mathbf{T} .
$$

In the above equations, $P$ and $\mu$ are the fluid pressure and dynamic viscosity, respectively, $w$ is the vertical (i.e., out-of-plane) component of the velocity vector, $\mathbf{u}, \mathbf{n}$ is the normal vector, and $\mathbf{T}$ is the surface traction, which incorporates surface tension. Finally the stress tensor $\tau$ is defined as

$$
\tau=-P I+\mu\left(\nabla \mathbf{u}^{T}+\nabla \mathbf{u}\right),
$$

in which $I$ denotes the identity matrix and superscript $T$ denotes matrix transpose. On the lateral edges of the thin film, the azimuthal velocity, $v$, must match the velocity of the inner or outer cylinder by the no-slip condition. Thus

$$
\left.v\right|_{r=R_{i}}=\Omega R_{i} \quad \text { and }\left.\quad v\right|_{r=R_{o}}=0,
$$

where $R_{i}$ and $R_{o}$ are defined in Figure 1(b) and the angular speed $\Omega$, also defined in Figure 1(b), is expressed in $\mathrm{rad} / \mathrm{s}$. In addition, the film is assumed to satisfy clamped boundary conditions so that $H$ and its radial derivative, $H_{r}$, vanish along $r=R_{i}$ and $r=R_{o}$.

A numerical solution of a set of equations very similar to (2.1) through (2.5) is presented in Slim et al., ${ }^{1}$ but the method, which employs a Chebyshev pseudo-spectral discretization, is nontrivial. In any event, we seek a solution of a lower-order set of equations, derived under the slender geometry approximation outlined in Sec. I. As shown in the rigorous derivation of Howell, ${ }^{3}$ the major simplifications are that out-of-plane velocities are much larger than in-plane velocities and that the film thickness, $h$, is constant, i.e., stretching is absent and the deflections to the upper and lower surfaces of the thin film are sinuous in nature (Figure 1(c)). Following Howell's approach (see also Appendix A of Teichman ${ }^{4}$ ), one can derive, after much algebra, the (linearized) fluidic Föppl-von Kàrmàn equation applicable to the geometry of Figures 1(b) and 1(c). This reads

$$
2 \mu h\left(\frac{H_{r \theta}}{r}-\frac{H_{\theta}}{r^{2}}\right)\left(v_{r}-\frac{v}{r}\right)-\rho^{-} g H+\left(\gamma^{+}+\gamma^{-}\right) \nabla_{H}^{2} H=\frac{1}{3} \mu h^{3} \nabla_{H}^{4} H_{t},
$$

where $g$ is gravitational acceleration, $\gamma^{+}$and $\gamma^{-}$denote, respectively, the surface tension along the upper and lower boundaries of the film, $\nabla_{H}^{2}$ is the horizontal Laplacian and subscripts indicate derivatives with respect to the spatial coordinates and time. In (2.6), the azimuthal velocity is given by

$$
v=\frac{\Omega R_{i}^{2}}{R_{o}^{2}-R_{i}^{2}}\left(\frac{R_{o}^{2}}{r}-r\right) .
$$

Combining (2.6) and (2.7) yields

$$
\frac{4 \mu h}{r^{3}} \cdot \frac{\Omega R_{i}^{2} R_{o}^{2}}{R_{o}^{2}-R_{i}^{2}}\left(\frac{H_{\theta}}{r}-H_{r \theta}\right)-\rho^{-} g H+\left(\gamma^{+}+\gamma^{-}\right) \nabla_{H}^{2} H=\frac{1}{3} \mu h^{3} \nabla_{H}^{4} H_{t},
$$

which is the axisymmetric analogue of (3.5) of Slim et al. ${ }^{1}$ Term 1 from the left-hand side specifies the out-of-plane motion due to in-plane stresses; compressive and tensile stresses drive and oppose buckling, respectively. Terms 2 and 3 reflect the influence of gravity and surface tension while the right-hand side term specifies the viscous bending resistance. 
In the analogous elastic membrane problem studied, for example, by Balmforth et al., ${ }^{9}$ the bending resistance term is of similar form, however, the Laplacian operator acts on $H$ rather than its time derivative. This seemingly trivial modification has substantial implications: when drawing the neutral stability curve of vanishing growth rate, $H_{t} \equiv 0$. Consequently the bending resistance term disappears in the problem of a viscous film but not so in the case of an elastic membrane. Note that the above comments apply whether $\nabla_{H}$ is defined in a rectilinear or an axisymmetric geometry.

To make further progress, it is helpful to non-dimensionalize (2.8) scaling horizontal lengths with the outer radius $R_{o}$ and vertical lengths with the (constant) film thickness $h=\varepsilon R_{o}$ where $\varepsilon \ll$ 1. Furthermore, we scale time, $t$, with the characteristic timescale $T$. Obvious choices for $T$ are $\Omega^{-1}$ or $(h / g)^{1 / 2}$, however, these selections introduce difficulties for small $\Omega$ or very thin films; as such, we take $T=\left(R_{o} / g\right)^{1 / 2}$.

In non-dimensional form (2.8) can be rewritten as

$$
\frac{12 \Omega T}{r^{3}} \cdot \frac{\beta^{2}}{1-\beta^{2}}\left(\frac{H_{\theta}}{r}-H_{r \theta}\right)-\frac{3 \Upsilon_{1} H}{\varepsilon}+\frac{3 \Upsilon_{2}}{\varepsilon} \nabla_{H}^{2} H=\varepsilon^{2} \nabla_{H}^{4} H_{t},
$$

where $r$ and $H$ are now non-dimensional variables and the geometric parameter $\beta$ gives the ratio of radii, i.e., $\beta=R_{i} / R_{o}(<1)$. Moreover the non-dimensional numbers $\Upsilon_{1}$ and $\Upsilon_{2}$ are defined by

$$
\Upsilon_{1}=\frac{\rho^{-} g R_{o} T}{\mu} \quad \text { and } \quad \Upsilon_{2}=\frac{\left(\gamma^{+}+\gamma^{-}\right) T}{\mu R_{o}},
$$

i.e., $\Upsilon_{1}$ and $\Upsilon_{2}$ measure the respective contributions of gravity and surface tension relative to the film viscosity.

We seek axisymmetric normal mode solutions of (2.9) that satisfy the clamped boundary conditions

$$
\left.H\right|_{r=\beta}=\left.H\right|_{r=1}=0,\left.\quad H_{r}\right|_{r=\beta}=\left.H_{r}\right|_{r=1}=0
$$

and are of the form

$$
H(r, \theta, t)=f(r) \mathrm{e}^{\sigma t+\mathrm{i} m \theta}+\text { c.c. },
$$

in which $\sigma$ is the non-dimensional (linear) growth rate associated with azimuthal mode number $m$ and c.c. denotes the complex conjugate. (We verify in Appendix A that $\sigma$ must be a real number.) Applying (2.11) in (2.9) yields the following differential eigenvalue problem:

$$
\begin{aligned}
& \frac{12 \Omega T}{r^{3}} \cdot \frac{\beta^{2}}{1-\beta^{2}}\left(\frac{\mathrm{i} m}{r} f-\mathrm{i} m f_{r}\right)-\frac{3 \Upsilon_{1} f}{\varepsilon}+\frac{3 \Upsilon_{2}}{\varepsilon}\left(f_{r r}+\frac{1}{r} f_{r}-\frac{m^{2}}{r^{2}} f\right) \\
= & \varepsilon^{2} \sigma\left(f_{r r r r}+\frac{2}{r} f_{r r r}-\frac{1}{r^{2}} f_{r r}-\frac{2 m^{2}}{r^{2}} f_{r r}+\frac{1}{r^{3}} f_{r}+\frac{2 m^{2}}{r^{3}} f_{r}-\frac{4 m^{2}}{r^{4}} f+\frac{m^{4}}{r^{4}} f\right)
\end{aligned}
$$

(cf. Eq. 3.15 of Teichman ${ }^{4}$ ). We solve (2.12) by adapting the spectral algorithms discussed in Weideman and Reddy. ${ }^{10}$ Details are presented in Appendix B.

\section{B. Theoretical predictions}

By varying the independent non-dimensional parameters $\beta=R_{i} / R_{o}, \varepsilon=h / R_{o}, \Omega T, \Upsilon_{1}$, and $\Upsilon_{2}$, we can now predict, via the solution of (2.12), the most unstable azimuthal wave-number, $m^{\star}$, in different regions of parameter space. Sample model output is presented in Figure 2, which indicates the change of surface buckling pattern as $\beta$ is increased from $\frac{1}{5}$ to $\frac{4}{5}$. A more comprehensive result is that of Figure 3, which shows the variation of $m^{\star}$ with $\varepsilon$ and $\beta$ for different choices of $\Omega T, \Upsilon_{1}$, and $\Upsilon_{2}$. Note that $m^{\star}$ becomes indefinitely large as $\varepsilon \rightarrow 0$ and $\beta \rightarrow 1$. In the latter case, the gap width becomes vanishingly small so that the slender geometry approximation can no longer be applied with confidence. The former result can be understood by examining the right-hand side of (2.9): as $\varepsilon \rightarrow 0$, the bending resistance term quickly also tends to zero suggesting that the thin film can be readily deformed even at small azimuthal wavelength. Surface tension alone cannot resolve this degeneracy; as explained by Ribe,$^{11}$ the forces associated with surface tension and compression 

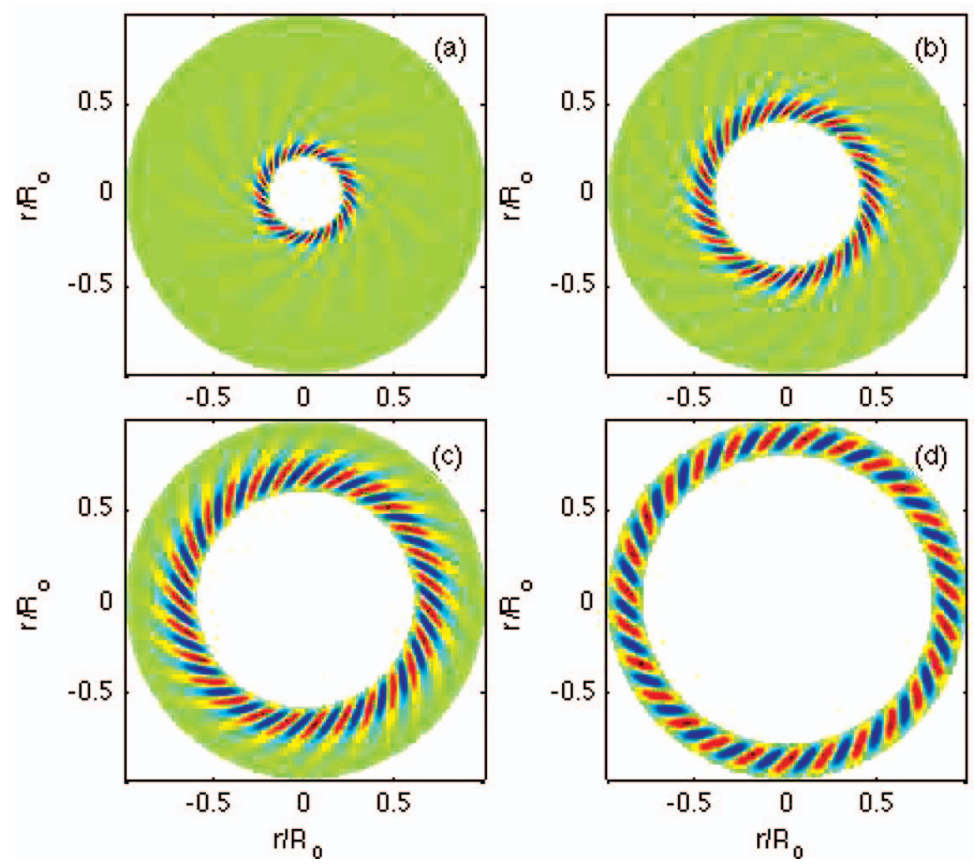

FIG. 2. Out-of-plane displacement field associated with the most unstable azimuthal mode of (2.12) for (a) $\beta=\frac{1}{5}\left(m^{\star}\right.$ $=16)$, (b) $\beta=\frac{2}{5}\left(m^{\star}=23\right)$, (c) $\beta=\frac{3}{5}\left(m^{\star}=27\right)$, and (d) $\beta=\frac{4}{5}\left(m^{\star}=27\right)$. Other parameter values are as follows: $\varepsilon=0.0207, \Omega T=0.365, \Upsilon_{1}=14.2$, and $\Upsilon_{2}=4.53 \times 10^{-3}$. The inner cylinder of Figure 1(b) occupies the white region in the center of each image and rotates in the counter-clockwise direction.

(a)

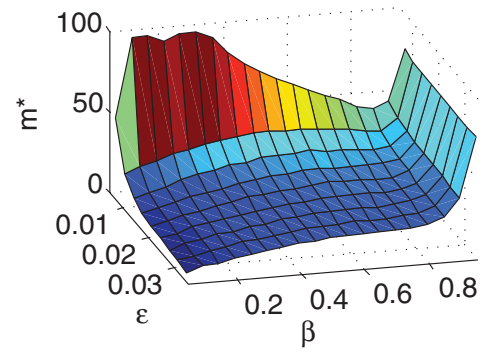

(c)

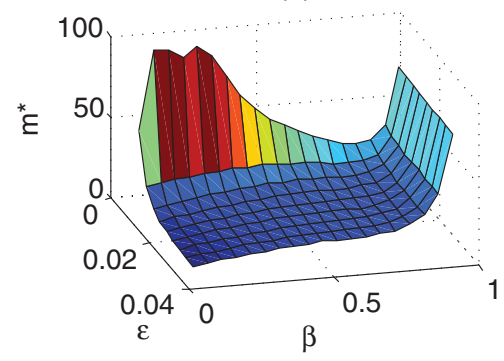

(b)

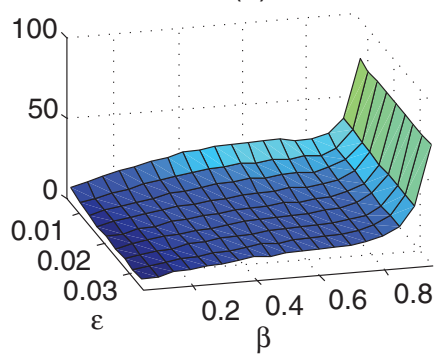

(d)

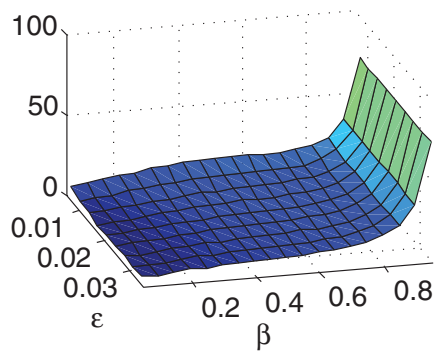

FIG. 3. Variation of $m^{\star}$ with $\varepsilon$ and $\beta$ as predicted by the numerical solution of (2.12). Note that the minimum value of $\varepsilon$ is $6.9 \times 10^{-3}$, not 0 . Parameter values are as follows: (a) $\Omega T=0.365, \Upsilon_{1}=14.2$, and $\Upsilon_{2}=4.53 \times 10^{-3}$; (b) $\Omega T=0.365$, $\Upsilon_{1}=5.93$, and $\Upsilon_{2}=1.89 \times 10^{-3}$; (c) $\Omega T=0.632, \Upsilon_{1}=8.22$, and $\Upsilon_{2}=7.85 \times 10^{-3}$; and (d) $\Omega T=0.632, \Upsilon_{1}=3.42$, and $\Upsilon_{2}=3.27 \times 10^{-3}$. Thus moving from the left-hand side panels to their right-hand side counterparts, we increase $\mu$ by a factor of 2.4 (see Sec. III). Likewise in moving from the upper panels to their lower counterparts, we decrease $g$ by a factor of 3 . 


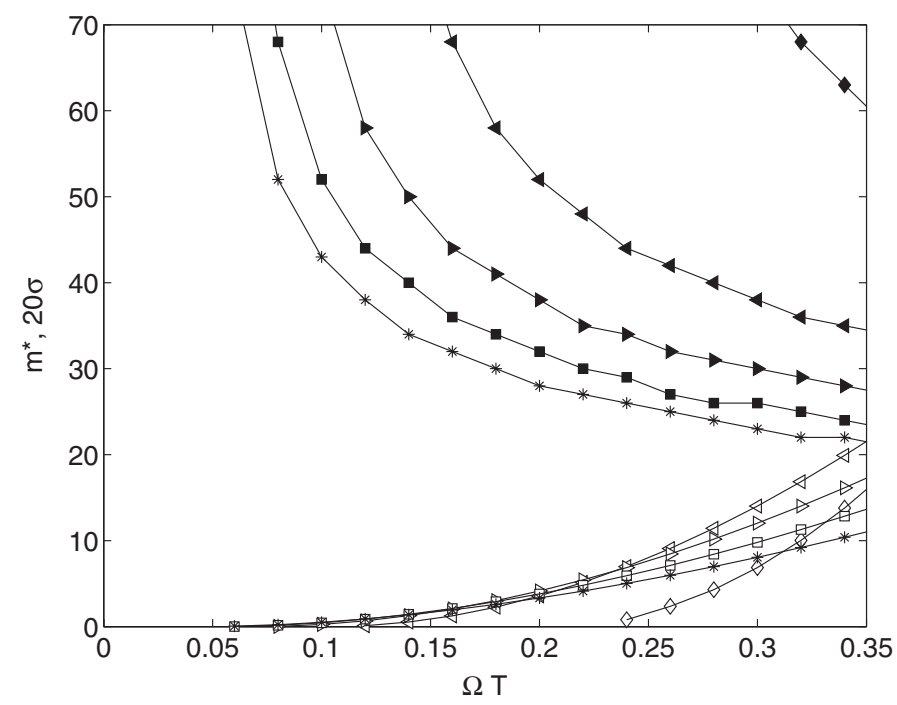

FIG. 4. Variation of $m^{\star}$ (solid symbols) and $\sigma$ (open symbols) with $\Omega T$ for $\beta=0.655, \Upsilon_{1}=14.2$, and $\Upsilon_{2}=4.53 \times 10^{-3}$ as predicted by the numerical solution of (2.12). Symbols are as follows - diamonds $(\diamond): \varepsilon=6.90 \times 10^{-3}$, left-facing triangles $(\triangleleft): \varepsilon=0.0138$, right-facing triangles $(\triangleright): \varepsilon=0.0207$, squares $(\square): \varepsilon=0.0276$, and stars $(*): \varepsilon=0.0345$.

both scale as 1/wavelength squared. Consequently, and whether one examines an axisymmetric or rectilinear flow, "if [compression] exceeds surface tension for some wavelength (as it must if buckling is to occur) then it will do so for all wavelengths."

Figure 4 shows that an even more severe degeneracy arises for small $\Omega T$ in which case $m^{\star}$ becomes arbitrarily large irrespective of $\varepsilon$. Consistent with the previous discussion, this prediction is likewise unreasonable: the asymptotic results (2.9) and (2.12) are long wave equations, i.e., they assume that the thin film has small curvature, which cannot be satisfied for large $m^{\star}$ (Benjamin and $\operatorname{Mullin}^{8}$ ). Moreover, on physical grounds, we expect there to exist a finite critical forcing below which film buckling does not occur in the same way that, for example, Rayleigh-Bénard convection cells cannot develop when the Rayleigh number associated with free-boundary convection is less than $27 \pi^{4} / 4$ (Drazin and Reid ${ }^{12}$ ). Even so, there exists a "silver lining" that has, to the best of our knowledge, been overlooked in previous commentary on this topic. Figure 4 also shows curves of the non-dimensional linear growth rate, $\sigma$. In contrast to $m^{\star}$, which becomes arbitrarily large as $\Omega T \rightarrow 0, \sigma$ becomes indefinitely small suggesting that the associated buckling instabilities, though present, grow extremely slowly. Complementing this observation, Figure 5 indicates curves of constant $\sigma$ (including $\sigma=0$ ) in the $\Omega T$ - $m$ plane for prescribed $\beta, \varepsilon, \Upsilon_{1}$, and $\Upsilon_{2}$. Whereas curves with $\sigma>0$ exhibit a global minimum, the neutral stability curve is monotone decreasing with a lower bound of zero. So although (2.9) and (2.12) cannot reliably estimate the incipient point of buckling, there is reason to be optimistic that this equation can do an adequate job of predicting buckling patterns for larger $\Omega T$. Indeed in the rectilinear case, Slim et al. ${ }^{1}$ remark that "for small and moderate wave-numbers, the viscous plate model approximates the growth rate and mode structures of the full Stokes equations very well." Our method of corroboration is different. Rather than numerically solving (2.1) and (2.2), we juxtapose the predictions of (2.12) with analogue laboratory data. Comparisons are presented in Sec. IV A. First, it is necessary to describe the experimental apparatus and technique.

\section{EXPERIMENTAL PROCEDURE}

Laboratory experiments were conducted in an annular glass beaker of height $18.0 \mathrm{~cm}$. The inner radius of the outer cylinder $\left(R_{o}\right)$ measured $14.7 \mathrm{~cm}$ whereas the outer radius of the inner cylinder measured $3.7 \mathrm{~cm}$. As shown schematically in Figure 6, a disk assembly constructed of stainless steel and consisting of a shaft, collar, disk, and perimetric ring was placed inside the beaker. The protruding 


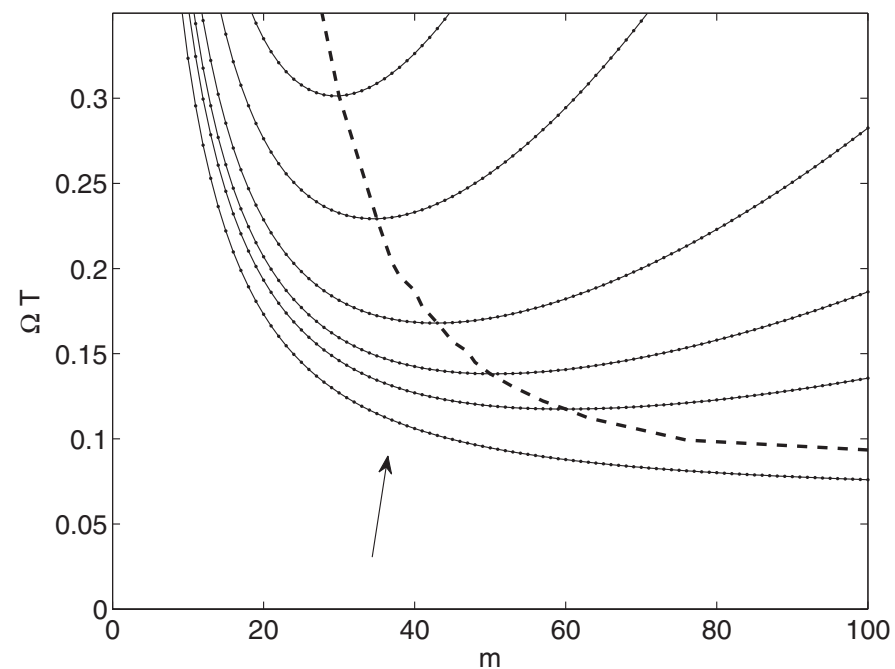

FIG. 5. Curves of constant (non-dimensional) growth rate with $\sigma=0,0.030,0.061,0.122,0.304,0.608$. The dashed curve connects the respective local minima and the arrow indicates the direction of increasing $\sigma$. Parameter values are as follows: $\beta=0.655, \varepsilon=0.0207, \Upsilon_{1}=14.2$, and $\Upsilon_{2}=4.53 \times 10^{-3}$.

shaft fit inside a lubricated brass groove, which was itself a component of a cylindrical base plate that formed a water-tight seal in the beaker annulus. When connected to a variable-speed DC electric motor (20 rpm, $45 \mathrm{rpm}$, or $90 \mathrm{rpm}$ Precision Gear Motor by Servocity), the disk assembly rotated at constant angular speed in the horizontal plane. Special measures were undertaken to minimize wobble (i.e., out of plane motion) of the disk assembly.

Three disk assemblies were employed where in each case the respective thickness of the disk and perimetric ring was $1.6 \mathrm{~mm}$ and $12.7 \mathrm{~mm}$. The purpose of the ring was to allow us to vary the film thickness, $h$, over a broader range of values than would have been possible had the disk alone been employed. (By contrast, if we had fabricated the disks out of $12.7 \mathrm{~mm}$-thick stainless steel, a non-trivial upgrade of motor size would have been necessary.) Relevant $\beta$ values for the disk assemblies are given in Table I.
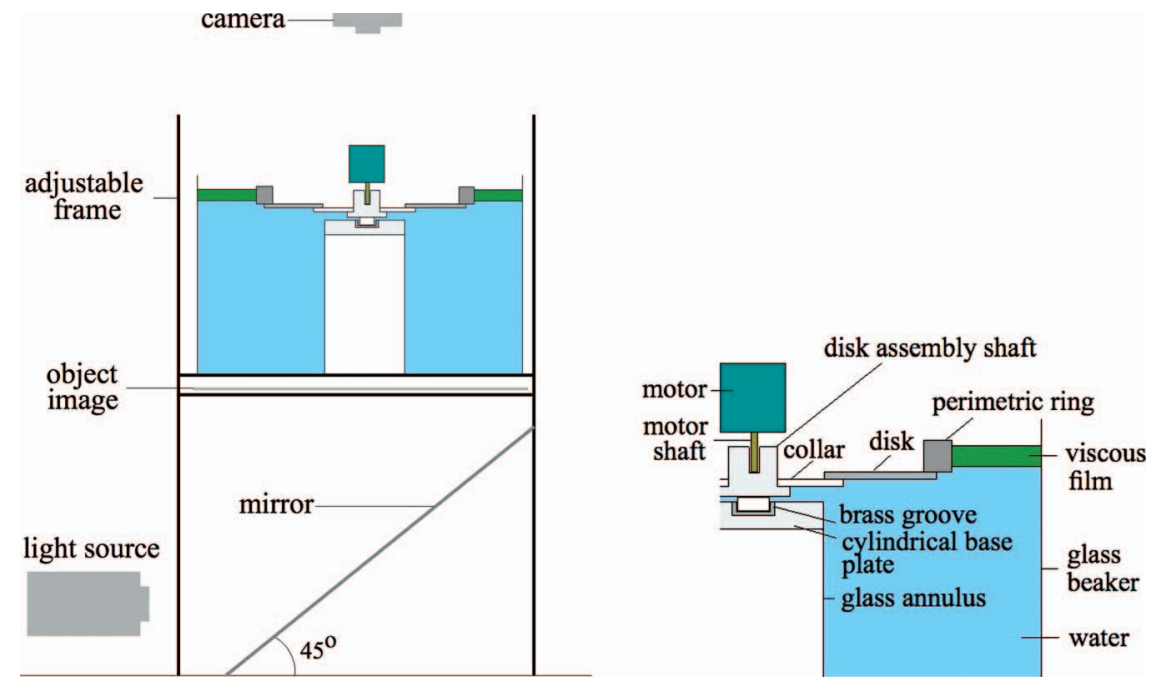

FIG. 6. (Left) Schematic of the experimental set-up (camera elevation and light source position not to scale). The beaker rests on an adjustable frame of T-slotted aluminum bars, i.e., 80-20 Inc., product 1010. (Right) Detailed schematic of the disk assembly. 
TABLE I. Geometric details for the disk assemblies. Measurements of $R_{i}$ are considered accurate to within $1 \mathrm{~mm}$.

\begin{tabular}{lcc}
\hline \hline Disk assembly & $\begin{array}{c}\text { Outer radius of the } \\
\text { perimetric ring, } R_{i}(\mathrm{~cm})\end{array}$ & $\beta=R_{i} / R_{o}$ \\
\hline $\mathrm{A}$ & 7.3 & 0.497 \\
$\mathrm{~B}$ & 9.8 & 0.667 \\
$\mathrm{C}$ & 12.4 & 0.844 \\
\hline \hline
\end{tabular}

In a typical experiment, the beaker was filled with warm tap water to a depth of approximately $14 \mathrm{~cm}$, so that the bottom of the perimetric ring was submerged to a depth of at least $2 \mathrm{~mm}$. Thereafter, the beaker was left undisturbed overnight; the following morning, bubbles of previously dissolved air were scraped off the side walls and bottom of the beaker. A relatively small quantity of viscous oil, measured by mass using an Acculab Vicon VIC212 balance and having a specific gravity of 0.97, was then poured into the water. The system was left undisturbed for an additional eight or more hours after which a thin film with a smooth free-surface was observed. The film thickness was between $1.9 \mathrm{~mm}$ and $5.0 \mathrm{~mm}$ with a measurement uncertainty of approximately $0.1 \mathrm{~mm}$. Films with $h \lesssim 2 \mathrm{~mm}$ (i.e., $\varepsilon \lesssim 0.014$ ) could rupture easily while those with $h \gtrsim 5 \mathrm{~mm}$ (i.e., $\varepsilon \gtrsim 0.034$ ) often produced an irregular buckling pattern. Two different viscosities of oil were used in our experiments, namely $v=12500 \mathrm{cSt}$ and $v=30000 \mathrm{cSt}$ where $v$ is the kinematic viscosity. Oils were silicone-based and manufactured by Xiameter (PMX 200 oil category). Product information supplied by Xiameter indicates that $\gamma^{+}=0.022 \mathrm{~N} / \mathrm{m}$ and $\gamma^{-}=0.044 \mathrm{~N} / \mathrm{m}$.

Our optical set-up consisted of an 8-bit high-speed camera (RedLake, MotionPro), a light source (Reflecta Diamator A slide projector), a mirror angled at approximately $45^{\circ}$ and an object image consisting of a regular array of horizontal and vertical lines with a line thickness of $1 \mathrm{~mm}$ and a center-to-center line spacing of $4 \mathrm{~mm}$. The camera was mounted on a tripod and centered about the (vertical) axis of the disk assembly. The camera lens (Nikon $35 \mathrm{~mm}$ ) sat approximately $80 \mathrm{~cm}$ above the free-surface of the thin film. Because a portion of the thin film was obscured by a clamp holding the motor in place, experimental images were cropped to reveal just over one-half of the free-surface (see Figure 7 below).

Experiments were begun by slowly increasing the angular speed of the motor to a prescribed value of between $0.5 \mathrm{rad} / \mathrm{s}$ and $4.3 \mathrm{rad} / \mathrm{s}$. (Representative Reynolds numbers for the less and more viscous silicone oils were 1.0 and 0.25 , respectively, where the Reynolds number is defined as $\operatorname{Re}=\Omega R_{i}\left(R_{o}-R_{i}\right) / \nu$.) Once transients had subsided and a regular wave field was observed, the camera began recording images at a frame rate of $100 \mathrm{fps}$ over a $15 \mathrm{~s}$ to $20 \mathrm{~s}$ time interval. A representative image from the laboratory is shown in Figure 7. Although motion in the thin film ceased very shortly after the disk assembly stopped rotating suggesting, consistent with the assumptions of Sec. II, small fluid inertia, at least 30 min elapsed between successive experimental runs to allow the thin film to return to its quiescent and smooth initial state.

So as to reduce errors in experimentally estimating $m^{\star}$, pairs of images taken in a short time (i.e., $0.02 \mathrm{~s}$ to $0.04 \mathrm{~s}$ ) apart were subtracted one from the other (Figure 7). The change in the distortion pattern of the object image then allowed us to more readily count the number of wave troughs or crests, for purposes of estimating $m^{\star}$, or to track their evolution in time, for purposes of estimating the associated angular phase speed, $c_{p}$. Values for $m^{\star}$ reported below represent the average of approximately 15 such image subtractions. This information was in turn used to estimate $c_{p}$ : the ratio $c_{p} / \Omega$ equals $\mathcal{N}_{1} / m^{\star}$ where $\mathcal{N}_{1}$ is the number of wave troughs or crests passing a fixed point in the experimental image in one full rotation of the disk assembly.

When measuring wave amplitudes, the high speed camera described above was replaced with a LaVision Imager E-lite model with a Nikon $60 \mathrm{~mm}$ lens. This camera captured images at $15 \mathrm{fps}$ and was oriented so that it made an angle of approximately $30^{\circ}$ with respect to the (undisturbed) free-surface of the film. Moreover, we replaced the slide projector of Figure 6 with a verticallyoriented $5 \mathrm{~mW}$ Cemarline CL 801 laser pen (Cemar Electro Inc.) that emits light at $670 \mathrm{~nm}$. This particular laser pen was selected because the light intensity varies by only a small fraction along the 

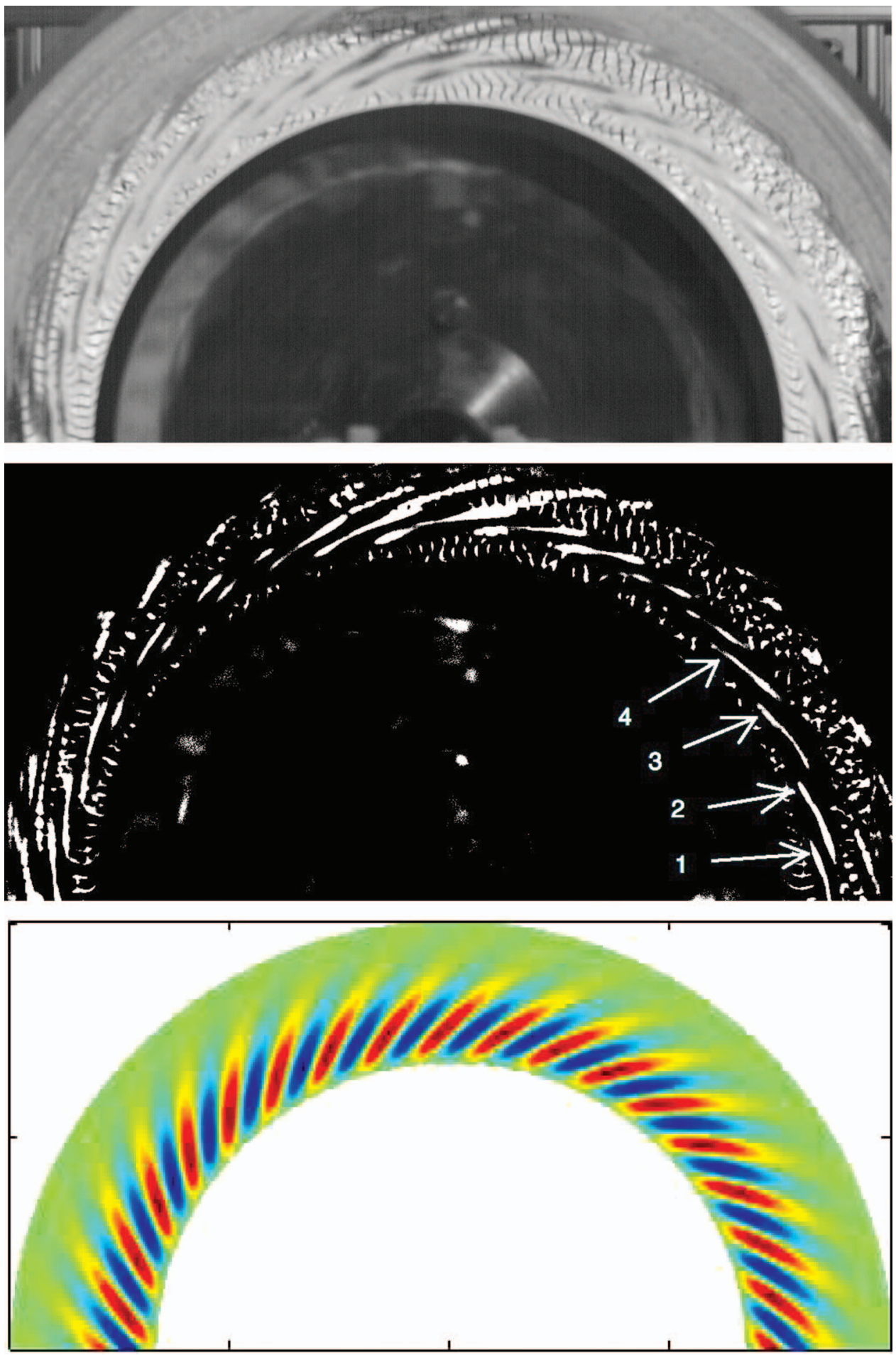

FIG. 7. Snapshot image from an experiment with $\Upsilon_{1}=14.5, \Upsilon_{2}=4.50 \times 10^{-3}$, i.e., $(v=12500 \mathrm{cSt}), \beta=0.667$, $\varepsilon=0.0193$, and $\Omega T=0.239$. (Top) Raw image and (middle) subtracted image. In the latter case, the arrows and numbers denote individual wave crests. For the sake of comparison, the bottom panel shows the associated numerical solution. 


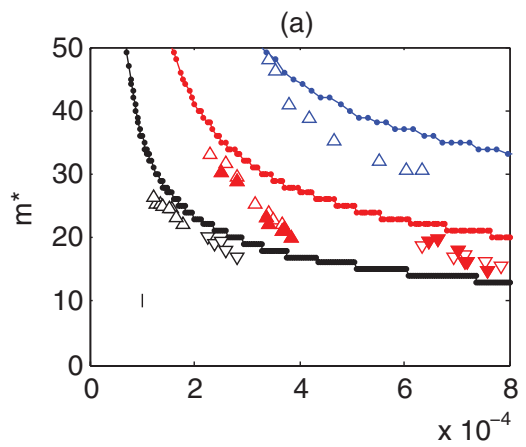

(c)

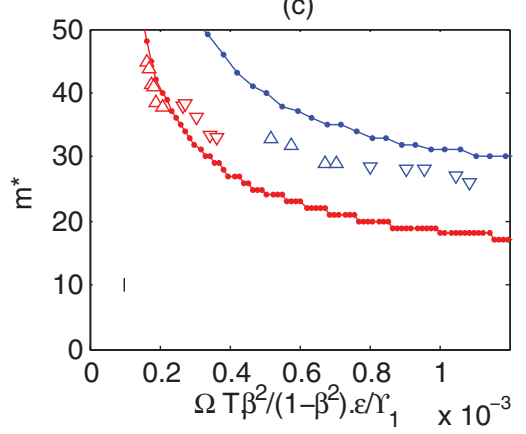

(b)

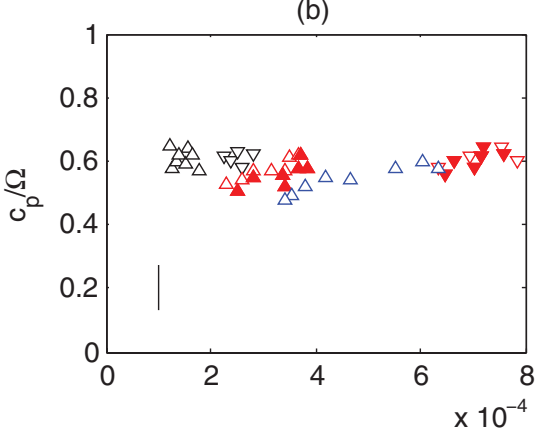

(d)

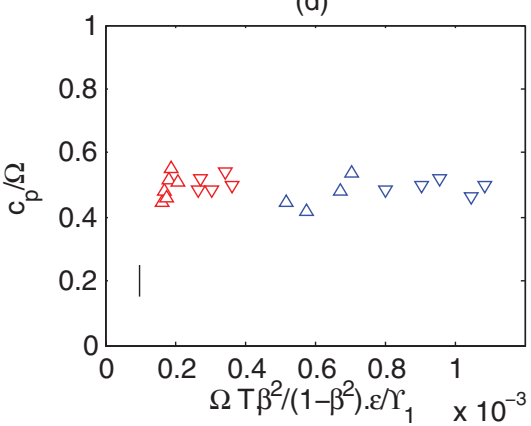

FIG. 8. (a) Comparison between the solution of (2.12) and experimental measurements for $v=12500 \mathrm{cSt}$. Black, red, and blue curves correspond, respectively, to $\beta=0.497,0.667$, and 0.844 ; in all cases, $\Upsilon_{1}=14.5$ and $\Upsilon_{2}=4.50 \times 10^{-3}$. (b) Measured angular phase speeds for $v=12500 \mathrm{cSt}$. Data points for panels (a) and (b) are summarized in Table II. (c) Comparison between the solution of (2.12) and experimental measurements for $v=30000 \mathrm{cSt}$. Red and blue curves correspond, respectively, to $\beta=0.667$ and 0.844 ; in both cases, $\Upsilon_{1}=6.05$ and $\Upsilon_{2}=1.88 \times 10^{-3}$. (d) Measured angular phase speeds for $v=30000 \mathrm{cSt}$. Data points for panels (c) and (d) are summarized in Table III. Representative vertical error bars are as indicated.

length of the emitted laser sheet. Therefore, when the laser sheet intersected the free-surface, a thin line of nearly uniform intensity was observed. The silicone oil being very nearly transparent, this line appeared to be very faint in the recorded images. Consequently the free-surface was seeded by hand with hollow spherical particles (Extendospheres TG Hollow Spheres) of mean specific gravity of $0.72 \pm 0.05$. Although $\rho_{\text {particles }}<\rho$, we observed that the simple act of shearing the oil film caused a non-trivial fraction of the particles to become trapped inside the oil layer, i.e., the particles did not all accumulate at the free-surface. The nominal diameter range of the particles was $10 \mu \mathrm{m}$ to $150 \mu \mathrm{m}$, however, according to the manufacturer's specifications, between $5 \%$ and $10 \%$ of the spheres were of diameter $>150 \mu \mathrm{m}$. The seeding density was selected so that the mass percent of the particles was less than $0.15 \%$ the mass of the silicone oil. We confirm in Figure 8(a) below that particle seeding does not substantially alter the properties of the silicone oil at least insofar as the parameters of interest here.

Determinations of the out-of-plane displacements were made by measuring the distortion of the laser line, which, in the absence of shear, was a straight line passing through the center of the disk assembly. In the camera's field of view, a succession of free-surface waves passed from right-to-left, growing to a maximum amplitude between the edge of the disk assembly and the inner wall of the glass beaker. So as to determine this maximum amplitude, hereafter referred to simply as the wave amplitude, a series of images, rendered initially using DaVis 8.0 software, were superposed in MATLAB for the purpose of visualizing the wave envelope. This composite image was thresholded and filtered using a two-dimensional median filter to remove signal noise. The free-surface elevation was then determined by estimating the point of transition between dark and illuminated segments of the thresholded image. (This last step involved fitting pixel intensity data from each column of the thresholded image using a high-order polynomial - details are summarized by Bhattacharya. ${ }^{13}$ ) Finally, reference was made to a calibration image consisting of a well-illuminated snapshot image 
TABLE II. Data points descriptions - Figures 8(a) and 8(b). In all cases, $\Upsilon_{1}=14.5$ and $\Upsilon_{2}=4.50 \times 10^{-3}$.

\begin{tabular}{|c|c|c|c|c|}
\hline Symbol & Color & $\beta$ & $\varepsilon$ & Notes \\
\hline Upward triangle $(\triangle)$ & Black & 0.497 & 0.0160 & $\begin{array}{l}\text { Data are an amalgam of three independent } \\
\text { trials with } \varepsilon=0.0150,0.0163 \text {, and } 0.0165\end{array}$ \\
\hline Downward triangle $(\nabla)$ & Black & 0.497 & 0.0238 & $\ldots$ \\
\hline Upward triangle & Red & 0.667 & 0.0199 & $\begin{array}{l}\text { Data are an amalgam of two independent } \\
\text { trials with } \varepsilon=0.0193 \text { and } 0.0209\end{array}$ \\
\hline $\begin{array}{l}\text { Upward triangle, } \\
\text { filled }\end{array}$ & Red & 0.667 & 0.0205 & $\begin{array}{l}\text { Control experiment: oil seeded with } \\
\text { spherical particles }\end{array}$ \\
\hline Downward triangle & Red & 0.667 & 0.0337 & $\ldots$ \\
\hline $\begin{array}{l}\text { Downward triangle, } \\
\text { filled }\end{array}$ & Red & 0.667 & 0.0331 & $\begin{array}{l}\text { Control experiment: oil seeded with } \\
\text { spherical particles }\end{array}$ \\
\hline Upward triangle & Blue & 0.884 & 0.0186 & $\begin{array}{l}\text { Data are an amalgam of three independent } \\
\text { trials with } \varepsilon=0.0182,0.0188 \text {, and } 0.0191\end{array}$ \\
\hline
\end{tabular}

of a ruler held in the plane otherwise occupied by the laser sheet; thus we were able to convert wave amplitudes measured in pixel counts to measurements in a physical length scale, i.e., $\mathrm{mm}$.

\section{RESULTS AND DISCUSSION}

\section{A. Comparison between linear theory and laboratory measurements}

Qualitatively speaking, Figure 7 suggests a relatively robust agreement between the model of Sec. II A and analogue laboratory images. A minor difference between the two concerns the angle between the wave crests and the disk assembly perimeter, which appears to be moderately overpredicted. Also, whereas both the top and bottom panel of Figure 7 show a thin region immediately adjacent to the disk assembly where, consistent with the clamped boundary condition (2.10), the surface is relatively smooth, the radial extent of this region appears to be under-predicted.

More quantitatively, Figure 8(a) presents a comparison between the solution of (2.12) and the corresponding experimental measurement of $m^{\star}$ for the lower viscosity (i.e., $v=12500 \mathrm{cSt}$ ) oil. Small closed circles and open triangles indicate theoretical and laboratory data, respectively. Conversely solid triangles show the results of a pair of control experiments in which the oil was seeded with the Extendospheres TG Hollow Spheres according to the protocol summarized in Sec. III. No measurements of wave amplitude were made in these cases; rather, the control experiments were run to verify that the mechanical properties of the oil do not change in a significant way due to particle addition.

Corresponding data with the higher viscosity silicone oil are presented in Figure 8(c). When $v$ $=30000 \mathrm{cSt}$, we were unable to generate a wave field of satisfactory regularity when using disk assembly A for which $\beta=0.497$.

In general, the quantitative comparison between theory and experiment in Figures 8(a) and 8(c) is favorable. Although for the lower viscosity oil, generally smaller deviations are observed when $\Omega$ is relatively small, no such trend is evident for the higher viscosity oil. In all but the case of the downward red triangles of Figure 8(c), the theoretical prediction matches or exceeds its experimental

TABLE III. Data points descriptions - Figures 8(c) and 8(d). In all cases, $\Upsilon_{1}=6.05$ and $\Upsilon_{2}=1.88 \times 10^{-3}$.

\begin{tabular}{lcccc}
\hline \hline Symbol & Color & $\beta$ & $\varepsilon$ & Notes \\
\hline Upward triangle $(\triangle)$ & Red & 0.667 & 0.0130 & $\ldots$ \\
Downward triangle $(\nabla)$ & Red & 0.667 & 0.0201 & $\ldots$ \\
Upward triangle & Blue & 0.884 & 0.0208 & $\ldots$ \\
Downward triangle & Blue & 0.884 & 0.0283 & $\ldots$ \\
\hline \hline
\end{tabular}


counterpart, however, considering all the data of Figures 8(a) and 8(c) the degree of under- or overprediction is, in aggregate, less than $14 \%$ and nowhere exceeds $30 \%$. The comparison constitutes a non-trivial improvement over that reported in Figure 3-5(a) and 3-7 of Teichman. ${ }^{4}$ There exist at least two possible explanations for this difference. Although the experiments are relatively simple to run, ours have the chronological benefit of using present-day state-of-the-art flow visualization hardware and software, which may render our measurements slightly more accurate. Also, a number of Teichman's equations (e.g., his F.3) contain typos; it is unclear whether these were introduced during typesetting or at some earlier derivational stage.

Note, moreover, that the domain of each experimental data set indicates, for the prescribed value of $\varepsilon$, the range of $\Omega$ over which a regular buckling pattern of the type indicated in Figure 7 is observed. For $\Omega$ larger than or smaller than the values in this interval, the free-surface is, respectively, irregular and smooth, at least to the precision offered by our visualization equipment. Of course, the corresponding theoretical curves are not so versatile: for reasons alluded to above, they erroneously predict an unstable film even for small forcing. Moreover, they cannot anticipate the nonlinear effects that lead to a tangled wave field for large $\Omega$. On the basis of the comparison between theory and experiment, Figure 8 thereby offers a concise, graphical means of evaluating where the predictions associated with the Föppl-von Kàrmàn equation are reasonable or unreasonable in the fluidic-context.

The right-hand side panels of Figure 8 show measurements of the angular phase speed of the free-surface waves. Here no comparison with theory is possible because, as explored more exhaustively in Appendix A, the factor $\sigma$ that appears in (2.11) and (2.12) is a real, not a complex, number. This result is not an accident of the particular configuration considered in Figure 1: entirely equivalent results would apply if, as in the experiments of Suleiman and Munson, ${ }^{2}$ the inner cylinder were stationary while the outer cylinder were made to rotate or indeed if both cylinders rotated whether or not in the same direction. Even more generally and due to the self-adjoint nature of the governing equation and the associated clamped boundary conditions, $c_{p}=0$ in the related rectilinear problem studied by Slim et al. ${ }^{1}$ They note that the governing equation (i.e., the rectilinear analogue of Eq. (2.9)) is devoid of advective terms and is not therefore frame invariant. On physical grounds, however, the buckling pattern "cannot be biased by either bounding wall" (Slim et al. ${ }^{1}$ ): if one of the (planar) boundaries were fixed while the other translated at a fixed speed $U$, the resulting phase speed would be $c_{p}=\frac{1}{2} U$. Consistent with the axisymmetric experiments of Suleiman and Munson, ${ }^{2}$ generally comparable results are achieved here (i.e., $c_{p} / \Omega \simeq 0.5$ ).

\section{B. Wave amplitudes}

Ribe's ${ }^{11}$ discussion of Slim et al. ${ }^{1}$ highlights the need for experimental measurements of wave amplitudes. In this spirit, we present in Figure 9 laboratory data obtained using the procedure outlined in Sec. III. Consistent with the exposition of supercritical bifurcations presented in Sec. 49.1 of Drazin and Reid, ${ }^{12}$ the normalized wave amplitude, $\mathcal{A} / h$, increases with the applied shear rate, $\Omega T$. However, in both panels of Figure 9, a sudden increase in $\mathcal{A}$ is noted over a narrow band of $\Omega$. This interval represents a limited region of bi-stability in that waves of either small or large amplitude may be realized. Indeed, experimental images show an irregular oscillation in time between the small and large amplitude waves, both of which are represented among the data of Figure 9.

Qualitatively, the observations of the previous paragraph are consistent with the investigation of Balmforth et al.,${ }^{9}$ which examined the buckling of a (rectilinear) elastic plate. In describing a similar region of bi-stability, they remark that "the higher...states gain stability before the lower branches lose stability, leading to a multiplicity of stable states at a given shear." Quantitatively, however, important differences arise: in the study of Balmforth et al., sudden changes in the out-of-plane displacement are accompanied by simultaneous variations in the planar expanse of a given wrinkle. Here, by contrast, and although we cannot in a single experimental run simultaneously measure $m^{\star}$ and $\mathcal{A}$, different behavior is believed to occur. Parametrically-speaking, the open circles of Figure 9(a) coincide very nearly with the upward red triangles of Figure 8(a). (By the same token, one may contrast, respectively, the open and closed circles of Figure 9(b) with the upward and 
(a)

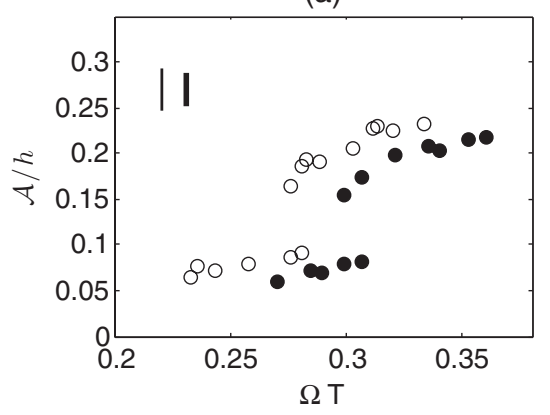

(b)

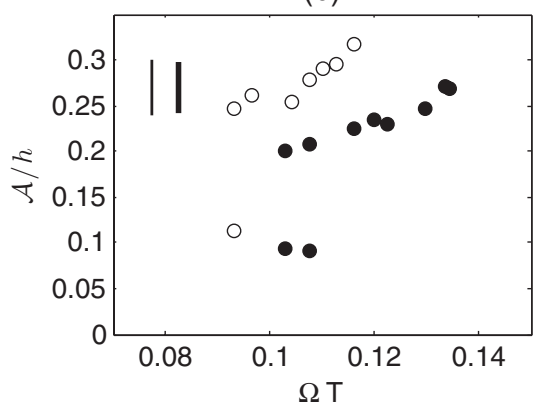

FIG. 9. Wave amplitude (normalized by $h$, the film thickness) vs. $\Omega T$. (a) $\Upsilon_{1}=14.5$ and $\Upsilon_{2}=4.50 \times 10^{-3}$ (i.e., $v$ $=12500 \mathrm{cSt}$ ); the open and closed circles correspond, respectively, to $\varepsilon=0.0214$ and $\varepsilon=0.0269$. (b) $\Upsilon_{1}=6.05$ and $\Upsilon_{2}$ $=1.88 \times 10^{-3}$ (i.e., $v=30000 \mathrm{cSt}$ ); the open and closed circles correspond, respectively, to $\varepsilon=0.0139$ and $\varepsilon=0.0205$. In all cases, $\beta=0.667$; the thin and thick solid vertical lines show representative vertical error bars for the open and closed circles, respectively. Note that wave amplitudes are too small to be reliably measured for small $\Omega T$, e.g., for $\Omega T \lesssim 0.23$ when considering the open circles of (a). Conversely the wave pattern becomes irregular for large $\Omega T$, e.g., for $\Omega T \gtrsim 0.14$ when considering the closed circles of (b).

downward red triangles of Figure 8(c).) In Figure 9(a), an abrupt increase in $\mathcal{A}$ is noted when $\Omega T \simeq 0.28$, however, the data of Figure 8(a) show no corresponding jump or drop in $m^{\star}$ when $\Omega T \cdot \beta^{2} /\left(1-\beta^{2}\right) \cdot \varepsilon / \Upsilon_{1} \simeq 3.1 \times 10^{-4}$. This latter observation is, of course, consistent with the trend of the analytical solution exhibited in Figure 8(a); being derived from a linear theory, however, and consistent with our previous remarks, this solution is unable to address any details regarding wave amplitudes including the possibility of multiple $\mathcal{A}$ for fixed $\Omega$. Although we defer to future studies a more detailed investigation of the bistable regime (including an analytical characterization of the stability limits associated with small and large amplitude waves), we may add the dissimilarity documented above to the list of non-trivial differences between the buckling of elastic membranes and viscous films.

A final remark concerning the data of Figure 9 is that the wave amplitude never exceeds approximately one-third of the film thickness. These are not the first experiments to reveal relatively modest out-of-plane displacements, which are likewise evident in Figures 15-17 of Taylor, ${ }^{14}$ for instance. It is interesting to note, however, that in obtaining the viscous plate model from the Stokes equations, the vertical displacement and layer thickness are assumed to be of comparable order (see, e.g., Sec. 4.2 of Howell $^{3}$ ). Thus the predictions of the fluidic Föppl-von Kàrmàn equation show positive agreement with laboratory measurement in a regime that is different from that assumed in the derivation of the lower-order equation. A pertinent question is whether analogous robustness is exhibited when the vertical displacement and layer thickness are closer together in magnitude. Unfortunately, this question cannot be adequately addressed here: whereas Figure 9 indicates that $\mathcal{A} / h$ increases as $\varepsilon$ decreases, there is a lower bound, imposed by capillarity, to the layer thickness. In other words and in the context of the previous experiments, $\mathcal{A} / h \sim 1$ requires an oil of high viscosity but low surface tension. Identifying such an oil and performing the requisite experiments is beyond the scope of the present inquiry.

\section{CONCLUSIONS}

An analytical and experimental study of the shear-induced buckling of a thin, viscous film in an axisymmetric geometry is presented. In-plane shear is generated by rotating the inner boundary relative to a stationary outer boundary, which results in buckling or out-of-plane displacements of the viscous fluid manifest as a regular pattern of free-surface waves.

Using the laboratory equipment described in Sec. III, we measured wave properties from digitized experimental images. Regarding as independent variables the gap width, the angular speed, $\Omega$, and the film thickness, $h$, and kinematic viscosity, $v$, we thereby determined the phase speed, $c_{p}$, 
and the number, $m^{\star}$, of waves excited. In the latter case, it is possible to compare laboratory data with predictions derived from a modified (or "fluidic") Föppl-von Kàrmàn model (Howell ${ }^{3}$ ), which has more traditionally been used to describe the wrinkling of elastic membranes (Landau and Lifshitz; ${ }^{7}$ Coman and Bassom ${ }^{15}$ ). The model equations yield a self-adjoint differential eigenvalue problem; numerical solutions are obtained using spectral methods in the manner of Weideman and Reddy. ${ }^{10}$ As exhibited by Figures 8(a) and 8(c), the agreement between theory and experiment is generally positive.

In contrast to Slim et al. ${ }^{1}$ who studied a similar problem but in a rectilinear geometry, we do not attempt to solve the full Stokes equations i.e., (2.1) and (2.2). In performing this (difficult) computation, Slim et al. ${ }^{1}$ determined that, in spite of degeneracies of the type exhibited in Figure 5 whereby $m^{\star} \rightarrow \infty$ at the point of neutral stability, there remains generally good agreement between solutions of the full model and its asymptotic analogue. Taken together, the results of this and the previous paragraph suggest that, provided none of $\Omega, h$ or the gap width is too small, asymptotic equations such as (2.9) may be more useful than is suggested by the discussion of, e.g., Benjamin and Mullin. ${ }^{8}$

Granted equations such as (2.9) cannot provide any information regarding $c_{p}$ nor the variation of the wave amplitude with $\Omega$, a deficiency that is here addressed by making additional measurements in the laboratory. Our amplitude data, summarized in Figure 9, reveal a limited region of bi-stability whereby waves of either small or large amplitude may be generated for the same $\Omega$. In contrast to Balmforth et al. ${ }^{9}$ however, there is evidence that the transition from small to large amplitude is not accompanied by a corresponding change in $m^{\star}$. To our best knowledge, these observations have not been made before in the context of thin film experiments of the type examined here.

We conclude by reiterating one of the important simplifying assumptions applied in this study, i.e., that the inner and outer boundaries are concentric so that the generated wave field is axisymmetric. A sensible, though non-trivial, extension of the present investigation, suggested by Professor N. J. Balmforth, would consist of breaking this symmetry so that the gap width varies with the cylindrical polar angle $\theta$. In this case, one would likewise expect potentially significant variations of the wave field morphology with $\theta$, e.g., waves of large and small amplitude, respectively, where the gap width is comparatively small and large. Whether the onset of buckling is dictated by the minimum or maximum gap width or some average of the two remains an open question to be addressed by future experiments.

\section{ACKNOWLEDGMENTS}

Financial support for this study was generously provided by Natural Sciences and Engineering Research Council (Canada) (NSERC) through the Discovery Grant and Research Tools and Instruments programs. The experiments of Sec. III benefitted from the helpful suggestions of J. A. Teichman and the fabrication assistance of the Technical Resources Group in the Department of Mechanical Engineering at the University of Alberta. The high speed camera used in the experiments was borrowed from the laboratory of Dr. David S. Nobes who provided additional experimental advice. INEOS Oligomers and the Kish Company kindly supplied free chemical samples. Finally, the thoughtful feedback provided by two anonymous referees helped to improve the clarity of our discussion.

\section{APPENDIX A: GROWTH RATE, $\sigma$}

By adapting the analysis of Southwell and $\operatorname{Skan}^{16}$ and Teichman, ${ }^{4}$ we demonstrate below that the growth rate, $\sigma$, defined in (2.11) must be real. Suppose first of all that

$$
H_{1}=\hat{H}_{1} \mathrm{e}^{\mathrm{i} \mathcal{P}_{1} t}
$$

is a solution of (2.9) where $\hat{H}_{1}$ is a complex function of $r$ and $\theta$ and

$$
\mathcal{P}_{1}=\mathcal{P}_{1 r}+\mathrm{i} \mathcal{P}_{1 i}
$$


Because the coefficients of (2.9) are real, there exists a second solution of the form

$$
H_{2}=\hat{H}_{2} \mathrm{e}^{\mathrm{i} \mathcal{P}_{2} t},
$$

where $\hat{H}_{2}=\hat{H}_{1}^{\star}$, the complex conjugate of $\hat{H}_{1}$, and

$$
\mathcal{P}_{2}=-\mathcal{P}_{1 r}+\mathrm{i} \mathcal{P}_{1 i}
$$

The above discussion suggests that

$$
\begin{aligned}
\varepsilon^{2} \mathrm{i}\left(\mathcal{P}_{1} H_{2} \nabla_{H}^{4} H_{1}-\mathcal{P}_{2} H_{1} \nabla_{H}^{4} H_{2}\right) & =\frac{12 \Omega T}{r^{3}} \frac{\beta^{2}}{1-\beta^{2}}\left(\frac{H_{2} H_{1 \theta}}{r}-\frac{H_{1} H_{2 \theta}}{r}-H_{2} H_{1 r \theta}+H_{1} H_{2 r \theta}\right) \\
& +\frac{3 \Upsilon_{2}}{\varepsilon}\left(H_{2} \nabla_{H}^{2} H_{1}-H_{1} \nabla_{H}^{2} H_{2}\right),
\end{aligned}
$$

which is obtained by substituting (A1) and (A3) into (2.9) then performing a number of additional algebraic operations. Here, for notational economy, we have dropped the hats on $H_{1}$ and $H_{2}$. We integrate (A5) over planar area, i.e.,

$$
\iint_{A}(\cdot) \mathrm{d} A=\int_{0}^{2 \pi} \int_{\beta}^{1}(\cdot) r \mathrm{~d} r \mathrm{~d} \theta
$$

Thus, for example,

$$
\iint_{A}\left[\left(H_{2} \nabla_{H}^{2} H_{1}-H_{1} \nabla_{H}^{2} H_{2}\right)\right] \mathrm{d} A=\iint_{A}\left[\nabla_{H} \cdot\left(H_{2} \nabla_{H} H_{1}\right)-\nabla_{H} \cdot\left(H_{1} \nabla_{H} H_{2}\right)\right] \mathrm{d} A=0 .
$$

The former equality follows from integration-by-parts and the latter equality follows from application of the clamped boundary conditions (2.10). In like fashion, and considering the left-hand side terms of (A5), it can be shown that

$$
\iint_{A}\left(\mathcal{P}_{1} H_{2} \nabla_{H}^{4} H_{1}-\mathcal{P}_{2} H_{1} \nabla_{H}^{4} H_{2}\right) \mathrm{d} A=\left(\mathcal{P}_{1}-\mathcal{P}_{2}\right) \iint_{A} \nabla_{H}^{2} H_{1} \nabla_{H}^{2} H_{2} \mathrm{~d} A .
$$

Finally, in considering those terms from (A5) that are proportional to $\Omega T$, we find that

$$
\begin{gathered}
\iint_{A}\left[\frac{12 \Omega T}{r^{3}} \frac{\beta^{2}}{1-\beta^{2}}\left(\frac{H_{2} H_{1 \theta}}{r}-\frac{H_{1} H_{2 \theta}}{r}-H_{2} H_{1 r \theta}+H_{1} H_{2 r \theta}\right)\right] \mathrm{d} A \\
=12 \Omega T \frac{\beta^{2}}{1-\beta^{2}} \int_{0}^{2 \pi} \int_{\beta}^{1} \frac{1}{r^{2}}\left(H_{1 r} H_{2 \theta}-\frac{2}{r} H_{1} H_{2 \theta}-H_{1 \theta} H_{2 r}\right) \mathrm{d} r \mathrm{~d} \theta \\
=12 \Omega T \frac{\beta^{2}}{1-\beta^{2}} \int_{0}^{2 \pi} \int_{\beta}^{1}\left(\frac{H_{1} H_{2 \theta}}{r^{2}}\right)_{r} \mathrm{~d} r \mathrm{~d} \theta \\
=0 .
\end{gathered}
$$

In deriving the first two equations, we again apply integration-by-parts and also a periodicity condition, which stipulates that $H_{j}(r, 0)=H_{j}(r, 2 \pi)$ where $j=1,2$. The third equation follows from the clamped boundary condition (2.10).

Summarizing the results of (A6) through (A8) we conclude that

$$
\left(\mathcal{P}_{1}-\mathcal{P}_{2}\right) \iint_{A} \nabla_{H}^{2} H_{1} \nabla_{H}^{2} H_{2} \mathrm{~d} A=0 .
$$

Non-trivial solutions of (A9) require $\mathcal{P}_{1}=\mathcal{P}_{2}$ whereupon applying (A2) and (A4) yields $\mathcal{P}_{1 r}=0$, i.e., $\mathcal{P}_{1}=\mathcal{P}_{2}=\mathrm{i} \mathcal{P}_{1 i}$. Solutions of (2.9) must therefore be proportional to $\mathrm{e}^{-\mathcal{P}_{1 i} t}$, where $\mathcal{P}_{1 i}$ is a real number. Setting $\sigma=-\mathcal{P}_{1 i}$ yields the form of the normal mode solution suggested by (2.11).

Note that the above derivation corrects a minor, but confusing, error in Appendix D of Teichman, ${ }^{4}$ who erroneously surmised that $\mathcal{P}_{2}=\mathcal{P}_{1}^{\star}$. 


\section{APPENDIX B: NUMERICAL DETAILS}

Solutions to (2.12) are obtained by writing the equation in the form of the matrix eigenvalue problem $A \mathbf{f}=\sigma B \mathbf{f}$ where the matrices $A$ and $B$ are defined by

$$
\begin{aligned}
A= & \frac{12 \Omega T}{r^{4}} \cdot \frac{\beta^{2}}{1-\beta^{2}} \cdot \mathrm{i} m I-\frac{12 \Omega T}{r^{3}} \cdot \frac{\beta^{2}}{1-\beta^{2}} \cdot \mathrm{i} m D_{1}-\frac{3 \Upsilon_{1} I}{\varepsilon}+\frac{3 \Upsilon_{2}}{\varepsilon} D_{2} \\
& +\frac{3 \Upsilon_{2}}{\varepsilon r} D_{1}-\frac{3 \Upsilon_{2}}{\varepsilon} \cdot \frac{m^{2}}{r^{2}} I
\end{aligned}
$$

and

$$
B=\varepsilon^{2}\left(D_{4}+\frac{2}{r} D_{3}-\frac{1}{r^{2}} D_{2}-\frac{2 m^{2}}{r^{2}} D_{2}+\frac{1}{r^{3}} D_{1}+\frac{2 m^{2}}{r^{3}} D_{1}-\frac{4 m^{2}}{r^{4}} I+\frac{m^{4}}{r^{4}} I\right),
$$

in which $I$ is the identity matrix and $D_{1}$ through $D_{4}$ are, respectively, the first through fourth order Chebyshev differentiation matrices (Weideman and Reddy ${ }^{10}$ ). The annular gap width $\beta \leq r \leq 1$ is mapped to the canonical interval $[-1,1]$ by introducing a new radial variable $\rho$ defined as

$$
\rho=\frac{2 r-1-\beta}{1-\beta} .
$$

The clamped boundary conditions now require

$$
\left.f\right|_{\rho=-1}=\left.f\right|_{\rho=1}=0,\left.\quad f_{\rho}\right|_{\rho=-1}=\left.f_{\rho}\right|_{\rho=1}=0,
$$

where $f_{\rho}=\frac{1}{2}(1-\beta) f_{r}$. Eigenvalues are determined using MATLAB's built-in function eig from which it is straightforward to determine the azimuthal wave-number with the largest associated (linear) growth rate for a given choice of $\beta, \varepsilon, \Omega T, \Upsilon_{1}$, and $\Upsilon_{2}$.

${ }^{1}$ A. C. Slim, J. Teichman, and L. Mahadevan, "Buckling of a thin-layer Couette flow," J. Fluid Mech. 694, 5-28 (2012).

${ }^{2}$ S. M. Suleiman and B. R. Munson, "Viscous buckling of thin fluid layers," Phys. Fluids 24(1), 1-5 (1981).

${ }^{3}$ P. D. Howell, "Models for thin viscous sheets," Eur. J. Appl. Math. 7, 321-343 (1996).

${ }^{4}$ J. A. Teichman, "Wrinkling and sagging of viscous sheets," Ph.D. dissertation, Massachusetts Institute of Technology, 2002.

${ }^{5}$ A. C. Slim, N. J. Balmforth, R. V. Craster, and J. C. Miller, "Surface wrinkling of a channelized flow," Proc. R. Soc. London, Ser. A 465, 123-142 (2009).

${ }^{6}$ A. E. H. Love, A Treatise on the Mathematical Theory of Elasticity (Dover, New York, 1941).

${ }^{7}$ L. D. Landau and E. M. Lifshitz, The Theory of Elasticity, 3rd ed. (Reed Educational and Professional Publishing Ltd., Oxford, 1986).

${ }^{8}$ T. B. Benjamin and T. Mullin, "Buckling instabilities in layers of viscous liquid subjected to shearing," J. Fluid Mech. 195, 523-540 (1988).

${ }^{9}$ N. J. Balmforth, R. V. Craster, and A. C. Slim, “On the buckling of elastic plates," Q. J. Mech. Appl. Math. 61, 267-289 (2008).

${ }^{10}$ J. A. C. Weideman and S. C. Reddy, "A Matlab differentiation matrix suite," ACM Trans. Math. Softw. 26, 465-519 (2000).

${ }^{11}$ N. M. Ribe, "All bent out of shape: Buckling of sheared fluid layers," J. Fluid Mech. 694, 1-4 (2012).

${ }^{12}$ P. G. Drazin and W. H. Reid, Hydrodynamic Stability (Cambridge University Press, Cambridge, 1981).

${ }^{13}$ S. Bhattacharya, "Buckling of a thin, viscous film in an axisymmetric geometry," Master's thesis, University of Alberta, 2012.

${ }^{14}$ G. I. Taylor, "Instability of jets, threads, and sheets of viscous fluid," in Proceedings of the 12th International Congress of Applied Mechanics, Stanford, 1968 (Springer-Verlag, Berlin, 1969), pp. 382-388.

${ }^{15}$ C. D. Coman and A. P. Bassom, "On the wrinkling of a pre-stressed annular thin film in tension," J. Mech. Phys. Solids 55, 1601-1617 (2007).

${ }^{16}$ R. V. Southwell and S. W. Skan, "On the stability under shearing forces of a flat elastic strip," Proc. R. Soc. London, Ser. A 105(733), 582-607 (1924). 